\title{
Les mots et la musique dans le drame shakespearien
}

\section{Pierre Iselin}

\section{(2) OpenEdition}

\section{Journals}

\section{Édition électronique}

URL : http://journals.openedition.org/shakespeare/1319

DOI : 10.4000/shakespeare.1319

ISSN : 2271-6424

Éditeur

Société Française Shakespeare

\section{Édition imprimée}

Date de publication : 1 novembre 1989

Pagination : 109-128

\section{Référence électronique}

Pierre Iselin, «Les mots et la musique dans le drame shakespearien », Actes des congrès de la Société française Shakespeare [En ligne], 7 | 1989, mis en ligne le 01 janvier 2007, consulté le 23 avril 2019.

URL : http://journals.openedition.org/shakespeare/1319; DOI : 10.4000/shakespeare.1319 


\title{
Société Française Shakespeare
}

\author{
Actes des Congrès \\ 1985 - 1986 - 1987
}

\section{LA FOLIE}

\section{SHAKESPEARE ET LES ARTS \\ LE TRAGIQUE}




\section{LES MOTS ET LA MUSIQUE DANS LE DRAME SHAKESPEARIEN}

On sait combien, dans 1'Europe de la Renaissance, le rapport de la langue à la musique a suscité de réflexions et de polémiques, et combien de recherches, archéologiques et théoriques au départ, comme celles de l'Académie de Baiff ou de la Camerata de Florence, ont abouti à des formes telles que la musique mesurée à l'ancienne, la monodie accompagnée, l'opéra même. Il est sans doute simpliste de présenter ainsi l'évolution de l'écriture musicale, de la polyphonie post-médiévale au style récitatif, comme la résultante du seul rapport au texte, placé sous la double exigence de l'intelligibilité et de l'expressivité. Mais on sait également comment les musiciens théoriciens de l'époque élisabéthaine (et jacobéenne), tels que Byrd, Morley, Campian et Dowland, ont tenté d'établir, parfois dans la controverse, une rhétorique musicale capable d'exprimer la «vie des mots» («the life of words», W. Byrd). Les mots des autres, s'entend, que le texte soit liturgique - le texte de l'ordinaire de la Messe, «revisité» par Byrd, est de ce point de vue un haut lieu de l'imagination musicale -, ou que le poème, en cette période de professionalisme naissant, soit dû à une autre main que celle du musicien. A la très remarquable exception de Campian, qui perpétue la tradition du poète-musicien dans la lignée des troubadours et de Guillaume de Machaut, les musiciens contemporains «déposent une musique le long de mots» filtrés par le poète, consentant celui-là : étroite palette lexicale, fréquence élevée d'onomatopées, évocation de sentiments extrêmes, recours à des mots-images, sont les ingrédients nécessaires au «word-painting» cher à Zarlino et à Morley. Cette vocation assez soudaine du discours musical à l'expressivité dramatique (voire à la théâtralité dans certaines formes avancées du madrigal), correspond en effet à la prolifération de techniques imitatives, jusque-là limitées aux chants d'oiseaux, aux bruits des villes et des batailles (Chant des Oiseaux, La Bataille, Les Embarras de Paris). La nouvelle école propose, outre ces procédés d'imitation naturaliste le recours à un cortège d'allégories plus ou moins codifiées, sur des mots-images (rise, fall, flow, down), l'utilisation presque systéma- 
tique du contraste homo-vs.-poly-phonie, les variations rythmiques - avec notamment la citation des rythmes de danse -, enfin la pratique régulière du chromatisme et de la dissonance (frottement de 7ème majeure, retards et fausses relations à des fins d'expressivité musicale.) L'imitation devient bientôt si caricaturale, ses effets si rhétoriques et ses figures si rapidement conventionnelles, que Campian y réagit sévèrement, reprenant pour dénoncer ces excès d'expressivité, l'image même du thêâtre :

But there are some, who to appeare the more deepe, and singular in their judgement, will admit no Musicke but that which is long, intricate, bated with fuge, chaind with sincopation, and where the nature of everie word is precisely exprest in the Note, like the old exploided action in Comedies, when if they did pronounce Memini, they would point to the hinder part of their heads, if Video, put their finger in their eye. But such childish observing of words is altogether ridiculous, and we ought to maintaine as well in Notes, as in action a manly cariage, gracing no word but that which is eminent, and emphaticall.

La référence à «every word... precisely exprest in Note» reprend presque littéralement un texte paru trois ans plus tôt sous la plume de Morley:

Dispose your music according to the nature of the words you are therein to express, or whatsoever matter it be which you have in hand, such a kind of music must you frame to $i^{2}$.

Shakespeare fait directement allusion à cette adéquation plus ou moins réussie du texte à la mélodie en au moins douze occurrences explicites : il y oppose cinq unités dénotant le langage (words, fréquence 7 ; ditty, fr. 4 ; matter, fr. 1 ; verse, fr. 1 ; et writing, fr. 1), aux deux seules unités musicales tune, fr. 7 et note, fr. 4 . Le même contraste est explicite dans des situations textuelles allusives, où l'air musical, conçu comme «timbre», est inapte à recevoir les paroles qu'on cherche à y adapter : c'est l'air de "Light o'love», notoirement dépourvu de l'équivoque "burden", que Lucetta ${ }^{3}$ juge trop «léger» pour y moduler la chanson grave de Protée, ou celui de "Greensleeves», impropre à recevoir les paroles du Psaume C. Mais c'est aussi sur le plan sémantique, l'extension de certaines unités spécifiquement musicales, comme "tune", "sound" et "song" vers des emplois non musicaux, qui tendent à 
valoriser la matière («matter»), c'est-à-dire le signifié, par rapport à son véhicule musical, proprement in-signifiant. Chez Hamlet, "tune", ici en emploi néologique, exprime l'illusion dérisoire du langage conventionnel :

Thus hath he... only got the tune of the time[...] which carries them through and through the most fanned and winnowed opinion...5.

La corrélation entre "sound" et "in-significance" participe de la vision nihiliste de Macbeth, pour qui

\section{Life $[. .$.$] is a tale$}

Told by an idiot, full of sound and fury, Signifying nothing.

Sur le mode sarcastique du fou, dans All's Well, la chanson ("song"), signe de mélancolie, devient monnaie d'échange :

I know a man who had this trick of melancholy sold a goodly manor for a song?.

"Tune", "sound" et "song" sont donc autant de néologismes (attestés par 1'O.E.D.) suscités par le débat très actuel sur les mots et la musique : la nonsémanticité du discours musical y est allusivement perçue comme in-signifiance, au double sens du terme.

Pourtant, connexe à la création musicale, se dessine une évolution de la terminologie, essentiellement forgée par la tradition polyphonique, vers un vocabulaire ou des emplois nouveaux, s'appliquant aux nouvelles données formelles et théoriques. Ainsi cohabitent, dans une polysémie encore floue, les concepts de modalité et de tonalité ("tone", "key"), de rythme et de mètre, de bourdon et de refrain - on peut noter, à ce propos, le néologisme Shakespearien sur "burden", qui étend la polysémie aux emplois du "bourdon" musical, jusque-là dénoté par "burdon". La palette shakespearienne dans le domaine musical est dotée d'une variété et d'une modernité étonnantes : en effet, plus de 280 bases lexicales, réparties sur plus de 2000 emplois, non compris les 371 emplois en indications scéniques, constituent un corpus suffisamment large pour étayer une étude systématique de la 
métalangue musicale - entendons par là le discours sur la musique. On observe ainsi conjointement une tendance caractéristique et constante à la néologie, et un rapport évolutif de l'allusion à la référence, mis en évidence par quatre traits sémantiques principaux.

1. D'abord, le discours, quoique majoritairement allusif dans l'ensemble du corpus, évolue vers un registre de plus en plus référentiel. Il commente une musique de scène en progrès constant.

2. Ensuite, la densité allusive croît avec le caractère spécifique du vocabulaire ; inversement, la plupart des acquisitions récentes sont employées en dénotation, alors que tous les emplois du vocabulaire théorique traditionnel sont situés en allusion :

- "air", néologisme musical attribué à Shakespeare, est dix fois sur dix utilisé en dénotation ;

- "burden" est régulièrement allusif au sens ancien de "cantusfirmus"; il est au contraire référentiel dans ses deux emplois au sens moderne de "refrain";

- Si "anthem" a deux occurrences métaphoriques qui font néologisme, c'est en raison de l'extension primitive très réduite de ce terme, compris comme univoque par un public qui assistait aux offices du Dimanche : Ici, la néologie, signalée par l'O.E.D. dans Two Gentelmen of Verona (sens n. 3) procède par élargissement («loosely'), non par scission ou fusion.

- Car, cet exemple excepté, la néologie musicale participe essentiellement d'un enrichissement de la polysémie, comme on l'a vu pour "song" "tune" et "sound" ainsi que d'une extension par emploi verbal de substantifs comme "voice", "trumpet" , "descant", "choir" et "bass" :

Methought the billows spoke, and told me of it

The winds did sing it to me; and the thunder,

That deep and dreadful organpipe, pronounc'd

The name of Prospero : it did bass my trespasss.

La métaphore cosmique d'Alonso est à cet égard très riche, en ce qu'elle met en parallèle le procès néologique ("bass" /' to bass')et le procès métaphorique des «signatures»"; elle inscrit en vertu de leur analogie une continuité entre tempête et apoplexie.

3. Parmi les emplois allusifs, on remarque, de plus, une désaffection progressive du jeu de mots au profit d'emplois métaphoriques, même si la distinction des deux registres est parfois difficile (dans l'exemple précédemment cité, "bass" 
est, intentionnellement ou non, homophone de "base").

4. Enfin, les grands passages à teneur musicale ${ }^{10}$ tendent à s'effriter en strettes dont la variété lexicale se resserre et dont, corrélativement, la répétition augmente.

Bien qu'il soit prématuré de tirer une conclusion de ces quelques observations, comment ne pas les mettre en regard de l'utilisation croissante des musiques de scène, qui apparaît sur le diagramme 1 ?

Comment ne pas céder à la tentation de voir l'équilibre se déplacer de l'allusion à la citation musicale ? Comment expliquer autrement que la pièce du corpus où l'on parle le plus sur la musique (c'est-à-dire The Taming of the Shrew) soit paradoxalement celle où la musique de scène, fort peu abondante, est l'une des moins significative.

On conçoit dès lors que la seule investigation du lexique est inapte à rendre intelligibles les rapports complexes des deux systèmes sémiotiques : «la musique, système fermé, qui est la même chose qu'il signifie, et le langage, système ouvert, qui n'est que l'expression de ce qu'il signifie, qui renvoie d̀ un sens qui lui est transcendant et dont il reçoit son unitê» ${ }^{11}$.

La clé de cette entreprise comparatiste nous semble inscrite dans les structures mêmes de l'épistémologie du XVIème siècle, où la notion de ressemblance surdétermine les rapports du signifiant au signifié, de la figure au pouvoir, de la similitude à l'identité. La «signature», définie par Foucault" comme «figure visible qui tire [la ressemblance] de sa profonde invisibilité», est le rapport entre la figure d'une plante et l'organe sur lequel elle a pouvoir, en vertu même de leur ressemblance. Relever l'analogie, c'est toutàla fois lever un des mystères du monde, c'est-à-dire faire avancer la connaissance, et en même temps s'emparer d'un pouvoir inscrit dans ce rapport secret. Ainsi la noix, qui selon Crollius ${ }^{13}$, «montre tout d fait le cerveau», prévient les maux de tête, par le même rapport d'analogie que la conjonction des astres «signe» le devenir des êtres. Ce concept véritablement central de la connaissance, troisième terme de la relation signifiant/signifié recouvrira pour nous les relations entre le mode musical et son pouvoir, la rhétorique et ses effets, et transitivement, les nombreuses analogies formelles, stylistiques, et théoriques, entre le système des sons et celui du sens. Car, à la Renaissance, comme l'a écrit également Foucault, «chercher le sens, c'est mettre au jour ce qui se ressemble. Chercher la loi du sens, c'est découvrir les choses qui sont semblables» ${ }^{14}$. C'est un $^{\text {' }}$ lieu commun que d'évoquer le caractère musical du langage, dans ses instances rythmique, ictique ou mélodique. La musicalité du vers shakespearien est un truisme, dont la réciproque est loin d'être la chose du monde la mieux partagée : car définir la musique comme langage, c'est hypostasier le signe en sens, et poser le 
problème en termes binaires et sémantiques, là où il est perçu comme herméneutique. La thêâtralité du monde évoquée par Jaques ${ }^{15}$, la lisibilité de la nature chantée par le Vieux Duc d'Arden ${ }^{16}$, participent de cette vision écrite, hiéroglyphique de la Création, où le mot comme le son ont pouvoir sur les êtres dans la mesure où ils sont manifestations explosées d'un Verbe initial. L'alchimiste de la Renaissance, se fondant sur ce rapport de similitude, fait des instruments de musique quil'entourent, de véritables instruments exploratoires, puisque c'est dans leur harmonie microcosmique que s'inscrivent les proportions (macro)cosmiques. La corde grave du luth, "Gamut"', «the ground of all accord», selon Hortensio ${ }^{17}$, est associée à la terre ; les sept rangs de cordes de la viole ou du luth sont la représentation musicale du système planétaire, comme les sept ouvertures du visage en sont la représentation corporelle, et les intervalles musicaux sont jusqu'à Mersenne le moyen analogique d'en calculer les rapports. C'est en ces termes quasi paracelsiens de redondance et de gémellité que s'explicite le concept d'harmonie universelle :

Such harmony is in immortal souls, But whilst this muddy vesture of decay Doth grossly close it in, we cannot hear it..$^{18}$

L'éloquence, qui, par la force des figures, a pouvoir sur l'âme, ne procède pas d'une autre «psycho-logie» que la musique, ordonnée, selon Platon", pour "ramener à l'ordre (catacosmesis) et à l'unisson (symphonia) les mouvements périodiques (de l'âme) qui se sont déréglés en nous». Dans ce système clos de la connaissance, dans cette «herméneutique de la ressemblance $»^{20}$, nul doute que les analogies de tous ordres entre le monde des sons, et celui des sens, entre le monde des instruments et celui des corps, n'aient fait l'objet de reconnaissance, voire de codification, d'abord peut-être dans une perspective d'efficacité, de «pouvoir». Sans entrer dans le détail de l'ésotérisme musical et des compositions orphiques qui font intervenir à la fois des données modales (éthiques, devrait-on dire, puisqu'il s'agit d'ethos) et des données astrales, mais sans non plus réduire la musique à cette seule récréation de l'esprit qu'évoque Lucentio dans The Taming of the Shrew ${ }^{21}$, c'est un topos que de dire avec Ronsard ${ }^{2}$ qu'elle «tire l' âme par l'oreille», ou avec Castiglione qu'elle «perce l' esprit» («perceth the mind») $)^{23}$. Pour être parodique, la remarque de Benedict (Much Ado) n'en fait pas moins référence à cette psychophysiologie classique de l'ex-stase, c'est-à-dire du «ravissement de l'âme»:

Now divine air! Now is his soul ravished!

Is it not strange that sheep's guts should hale

Souls out of men's bodies? ?24.

La synecdoque animale "sheep" $s$ guts' (pour strings), qui nous renvoie à 
la théorie déjà évoquée des «signatures», rappelle la décomposition presque scientifique des facteurs érogènes de l'aubade, selon Cloten :

Come on, tune: if you can penetrate her with your fingering, so: we'll try with tongue too : if none will do, let her remain it is a vice in her ears, which horse-hairs, and calves' guts, nor the voice of unpaved eunuch to boot, can never amend².

La réduction du charme (carmen) à sa composante animale est bien sûr intensifiée par le double-entendre particulièrement osé sur penetrate, fingering et tongue, et renforcé par la connotation sexuelle de "eunuch" ; elle fait aussi allusion à une série d'expériences, rapportées par Baptista Porta, au 16ème siècle, qui consistaient à tendre des instruments avec différentes natures de cordes : «celui qui alliait loup et mouton ne produisait aucune musique, tandis que le boyau de serpent, surtout celui de vipere, causait effroi et fausses couches ${ }^{26}$.

Le pouvoir musical, lié à un ethos modal ou instrumental, ne repose, comme le langage que sur la reconnaissance de signes, puisquec'est par le semblable que s'opère toute connaissance, que s'organise tout pouvoir, que s'effectue toute thérapie.

C'est par la gloutonnerie musicale qu'Orsino veut tuer sa maladie d'amour ; le redoublement est double, en l'occurrence, puisque c'est par le signe même de l'amour (à savoir la musique), qu'il veut gaver son mal jusqu'à l'excès, définissant ainsi une forme sans doute prophétique de mélothérapie homéopathique :

If music be the food of love, play on,

Give me excess of it, that, surfeiting,

The appetite may sicken, and so die ${ }^{27}$.

Ce qu'on pourrait appeler dans un raccourci ambigu «avalage sympathique», ou «engloutissement», Jaques le pratique pour exorciser son humeur bileuse : «I can suck melancholy out of a song, as a weasel sucks eggs».23. L'atrabilaire dissonant de la forêt d'Arden connaît alors une forme de bonheur : «here was he merry, hearing a song ${ }^{29}$. De cette antipathie éthico-humorale naît ironiquement, sur un autre registre de l'Instrument-monde, la dissonance des sphères :

If he, compact of jars, grow musical,

We shall have shortly discord in the spheres ${ }^{30}$. 
La signature musicale, qu'elle soit composante végétale ou animale de l'instrument, proportion harmonique d'un mode scellée par des rapports cosmiques, ou seulement timbre ou forme, donne du système musical une lecture assez transparente. Ainsi, «le mode triste et languissant $\rangle^{31}$ de la viole a sur la scène élisabéthaine une signification univoque : celle de l'harmonie ; par là, son éthos apollinien lui confere des pouvoirs curatifs et extatiques, voire résurrectionnels :

The still and woeful music that we have,

Cause it to sound, beseech you. [Music]

The viol once more ; how thou stirr'st, thou block!

The music there! $!^{32}$

Si la viole a le charme efficace de l'incantation magique, le luth, dont le mode est «prudent et modeste» ${ }^{33}$, a celui de l'élégie ; «lascif» pour Richard $\mathrm{III}^{34}$, mélancolique pour Falstaff, il est l'emblème même de la séduction, mais aussi du dépit amoureux :

Fals. 'Sblood, I am as melancholy as a gib cat, or a lugged bear. Prince. Or an old lion, or a lover's lute.

Fals. Yea, or the drone of a Lincolnshire bagpipe ${ }^{35}$.

L'éthique musicale des élisabéthains s'étend ainsi des «effets» orphiques de la viole ou guerriers du fifre, à ceux, diurétiques, de la cornemuse :

And others when the bagpipe sing i' th' nose, Cannot contain their urine... ${ }^{36}$

probable «sympathie» formelle entre la peau de l'instrument et la vessie. Des analogies ou isomorphismes de cet ordre déterminent la polysémie, plus ou moins teintée d'obscénité, de termes comme «fiddle», «stops», «ventages», «fiddlestick», «strings» ou «breath», contibuant à l'écriture d'un «corps musical», schématiquement évoqué par Du Bartas ${ }^{37}$, dans La Sepmaine (1581):

Notre langue est l' archet, notre esprit le sonneur, Nos dents, les nerfs battus, le creux de nos narines Le creux de l'instrument. 
Dans le drame shakespearien, la «signature» passe également de l'efficacité à l'image, du pouvoir à la métaphore, voire au jeu de mots : la langue devient trompette $^{38}$, les tendons des cordes ${ }^{39}$, la trachée-artère un tuyau d'orgue ${ }^{40}$, le corps féminin une viole de gambe :

You are a fair viol, and your sense the strings, Who finger'd to make man his lawful music

Would draw heaven down and all the gods to hearken, But being play'd upon before your time, Hell only danceth at so harsh a chime $e^{41}$.

Référence sexuelle encore plus appuyée chez Marlowe :

Like untun'd strings, all women are, Which long time untouched will harshly jar ${ }^{2}$.

La tenue de l'instrument entre les jambes, source classique de plaisanteries grasses, ses formes galbées, ses ouies latérales, la sculpture d'une tête de femme à l'extrémité du manche, sont autant de traits anthropomorphes d'une véritable fantastique musicale, où l'humain s'anastomose au musical, un peu à la manière de Jérôme Bosch ou d'Archimboldo.

Les représentations musicales du corps sont trop nombreuses et souvent trop longues pour être citées, ici ; du moins peut-on mentionner pour mémoire l'àpeu-près sur «heart/harp-strings ${ }^{43}$, l'analogie répétée entre la perte de la langue maternelle et un bel instrument sans cordes ${ }^{44}, l^{\prime}$ 'évocation musicale du dérèglement de Hamlet ou de Lear («untuned and jarring senses») ${ }^{45}$, les équivoques obscènes sur wind-instrument dans $O$ thello ${ }^{46}$, et surtout le long passage en double - voir triple entendre, où Hamlet s'identifie à un instrument doux et mélodieux, dont Guilderstern ne saura rien tirer :

Ham. $O$, the recorders /.../ Will you play upon this pipe ? I...।

Guild. I know no touch of it, my lord.

Ham. It is as easy as lying. Govern these ventages with your fingers and thumb, give it breath with your mouth, and it will discourse most eloquent music. Look you, these are the stops /...l 
Why, /... / you would play upon me, you would seem to know my stops, you would pluck the heart of my mystery, you would sound me from my lowest note to the top of my compass; and there is much music, excellent voice in this little organ, yet cannot you make it speak. 'Sblood, do you think I am easier to be played on than a pipe. Call me what you will, though you fret me, you cannot play upon $m e^{47}$.

L'imagerie de la flûte à bec (pipe, ventages, fingers and thumb, breath) glisse insensiblement vers celle du luth (pluck, fret) par le truchement d'une référence doublement ambiguë, «stops» ayant à la fois le sens obscène que l'on sait, et deux sens musicaux, celui de "trou" (synonyme de "ventages") pour les instruments à vent, et celui de «fret» pour les instruments à cordes (synonyme : "frets"). Ces vingt-trois emplois de vocabulaire musical tissent une polysémie à trois voix, complexe et parfaitement intégrée à la trame dramatique; entre les deux voix extrêmes, celle de la référence musicale pure - dont l'incohérence vient d'être soulignée - et celle de l'obscénité, apparaît la teneur du développement métaphorique : l'exil linguistique, ou condamnation au silence. La flûte à bec, référence scénique, devient figure oxymorique, instrument du silence, expression négative, comme en miroir, $d$ 'un monde où les mots sont «peints» et les langues bifides. On voit comment le discours de Mowbray ${ }^{48}$, dans lequel la métaphore musicale n'établissait que des relations bijectives et statiques entre le langage et les instruments a évolué chez Hamlet en complexité dramatique. Les démarches sont d'ailleurs inverses, l'une partant de la langue pour évoquer - sans référence - la viole et la harpe, l'autre prenant l'expérience référentielle - la flûte à bec des musiciens - pour support de son discours opaque. Les deux démarches se retrouvent, utilisées en alternance, dès les premières comédies (TGV et $S H R$ ), qui, faute d'être des comédies musicales, sont du moins celles où l'on parle le plus de musique. Le long dialogue en double-entendre de Julia et Lucette (acte I, scène 2, 79-98), qui fait en 19 lignes, 30 allusions à la musique polyphonique, a pour référence le billet de Protée et la gestuelle des sévices corporels traduits en altérations accidentelles (sharp $=$ diese $=$ pincement ; flat $=$ bémol $=$ gifle $^{49}$; inversement, au quatrième acte, Julia déchante sur une sérénade effectivement adressée sur scène à Sylvia (16 équivoques en 16 lignes, dont la polysémie "he plays false", 4.02.57, qui induit la polysémie de tout le passage). Dans $S H R$, la première leçon de luth ${ }^{50}$, qui tourne rapidement au pugilat, est rapportée par Hortensio :

Bap. Why then, thou canst not break her to the lute, Hor. Why no, for she hath broke the lute to me. 
De cette inversion des valeurs guerrières et musicales naissent des jeux de mots sur "frets" et "Jack» ( $a$ jack = un sautereau), qui n'ont ni la densité ni la complexité contrapuntique de la dispute Julia-Lucetta, bien que, elle aussi, ponctuée par des violences physiques.

La deuxième leçon de luth, au début du troisième acte, est quant à elle donnée effectivement sur scène ; elle est également objet de dispute entre les deux prétendants, Hortensio et Lucentio, et se conclut sur une allusion musicale au ménage à trois :

My lessons make no music in three parts ${ }^{\text {s1. }}$

La constante de tous les grands passages musicaux - qu'ils soient induits ou non par une musique de scène, que la musique soit la «teneur» ou le véhicule -, c'est la dissonance ou le contre-temps. Traitée sur le mode antiphrastique, l'allusion musicale participe d'une rhétorique de l'échec : il manque à la chanson de Julia un ténor, qu'elle ne s'attendait pas à entendre donner la sérénade à Sylvia; la chanterelle dissone pour Hortensio ; Kate a fait du luth un pilori, Hamlet de la flûte un tuyau muet, et Richard II perçoit dans les contre-temps d'une musique pourtant amie les syncopes de sa propre vie. L'accomplissement musical, qui échoue à séduire dans SHR ou CYM n'est pas mieux valorisé dans Twelfht Night, en la personne de Sir Andrew Aguecheek : «He plays o' th' viol-de-gamboys, and speaks three or four languages word for word without book»..."

L'association de la musique et de la langue rejoint implicitement le problème évoqué en préambule. Pour Mortimer d'ailleurs, le gallois qu'il ne comprend pas ne signifie rien d'autre que sa propre musique :

....thy tongue

makes Welsh as sweet as ditties highly penn'd, Sung by a fair queen in a summer's bow'r, With ravishing division to her lute ${ }^{53}$.

Le langage, qui ne renvoie ici à aucun sens, devient, comme la musique, un «système fermé». L'incompréhension prend le même signe, le même véhicule métaphorique, que d'autres formes perverties du langage, discordes privées et publiques; la sédition de la noblesse ( $\ll$ this jarring note of nobility» $)^{\text {s4 }}$ et la folie bruyante de Kate ${ }^{55}$ étant indistinctement traitées sur le mode de la dissonance. $L$ 'allusion musicale estalors expression sémantique du signe : le hautbois souterrain $\mathrm{d}^{\prime}$ Antony and Cleopatra ${ }^{56}$, est traduit par les soldats comme un présage funeste pour Antoine. Mais cette traduction devient trahison, ou du moins subversion, lorsque, dans Troïlus and Cressida, elle propose à un public, certes roué au double-entendre, plusieurs lignes de sens simultanées, dont la cohérence logique est souvent assurée 
par le discours occulte (érotique le plus souvent), alors que le discours de surface ne procède que par juxtaposition lexicale. Commentalors se risquer à la traduction d'un passage où ksing» module d'un sens érotique à l'autre (allure / coït with ${ }^{\text {s7) }}$ sous le sens légitime de "chanter" :

Ulysses She will sing (=allure) any man at first sign.

Thersites Any man can sing her if he can take her cliff; she's noted ${ }^{53}$.

Il est vrai que la polysémie du vocabulaire musical offre des possibilités comiques déjà naturellement nombreuses (par exemple bear the burden), auxquelles il faut ajouter les effets d'homophonie et d'à-peu-près (base/basse ; noting/nothing), et le vocabulaire connexe et tout aussi équivoque de la danse (brawl, measure...); The Taming of the Shrew et Two Gentlemen of Verona sont à cet égard le plus richement dotées : 22 "puns" musicaux pour la première, 14 pour la seconde, alors que Twelfth Night, souvent célébrée pour son humour musical, n'en compte que cinq, et The Tempest, deux. L'une des dernières strettes érotico-musicales est paradoxalement située dans une pièce, certes très équivoque, mais assez richement dotée en musique de scène (on observe en effet qu'à cette exception près, les pièces riches en musique de scène sont pauvres en jeux de mots musicaux, ainsi qu'en allusions «grasses») : Much Ado about Nothing. Ici, la chanson Light o' Love, déjà évoquée dans Two Gentlemen of Verona, est le point de départ d'un contre-point où le discours musical de surface n'est encore qu'un alibi ${ }^{39}$ :

Hero Why, how now ? Do you speak in the sick tune?

Beat. I am out of all other tune, methinks.

Marg. Clap's into 'Light o' Love' ; that goes without a burden. Do you sing it, and I'll dance it.

Beat. Ye light o' love with your heels ! Then if your husband have stables enough, you'll see he shall lack no barns ${ }^{60}$.

La séquence burden-love-dance... with your heels et l'équivoque barns/ bairns (children) est l'élément de cohérence entre images musicales hétérogènes. Les pouvoirs érogènes de la musique et de la danse donnent l'impression de contaminer la langue même qui s'y réfêre ; or, les registres de la technologie, de l'agriculture, du commerce ou de l'artillerie constituent un réservoir au moins aussi riche d'équivoques érotiques ${ }^{61}$. Pourtant, même si elle n'a pas le monopole de la paillardise, l'allusion musicale, comme la plus formellement symbolique des musiques de scène (que ce soit le Masque d'Hymen, dans As You Like It, ou le Masque de Céres dans La Tempête, ) est marquée par sa représentation mimétique de l'amour. 
«Musicke is a science of love matters occupied in harmonie and rythmos»". En définissant la musique comme une érotique appliquée à l'harmonie et au rythme, Morlcy anticipe sur un lieu commun de l'anthropologie moderne, qui considère la structure harmonique comme «érotique abstraite», réconciliation symbolique des contraires (la «science des contraires» de Boèce), et la structure rythmique comme épiphanie de la rythmique sexuelle ${ }^{63}$, traitée sur le mode comique dans des allusions comme «dance... with your heels», ou sur le mode ironique, lorsque le baiser musical de Desdémone et Othello donne naissance à une métaphore prophétique d'Iago : "I'll set down the pegs that make the music"s. Peut-être certains verront-ils dans l'oralité du baiser et sa relation imaginaire à l'artefact de l'instrument une relation entre le «corps archaïque» et le «corps musical» ${ }^{\circ}$ ? Il n'en reste pas moins que dans le drame, l'allusion d'Iago est le fil d'Ariane qui lie la mort d'Othello ("to die upon a kiss"66 à deux performances musicales - pratique peu fréquente dans la tragédie.

La première est l'aubade commandée par Cassio, qui, comme toute aubade ou sérénade sur la scène shakespearienne, de Titus Andronicus à Cymbeline, est vouée à l'échec : «as they say, to hear music, the general does not greatly care»". L'insensibilité à la musique de ce qui semble être des comemuses ${ }^{\text {a }}$, et le regret des musiques guerrières seraient-ils des signes avant-coureurs d'une brutalité animale ? En étouffant Desdémone, Othello ne cherche-t-il pas simplement à faire taire cette voix «capable de domestiquer un ours sauvage» (sing the savageness out of a bear)?

L'allusion musicale, comme trait de caractérisation, est encore plus cohérente dans la deuxième performance musicale, celle de la Chanson du Saule; emblème prémonitoire de la propre mort de Desdémone qui la chante, elle rappelle une autre mort où l'élément aquatique est véhiculé par l'image du cygne :

'Tis strange that death should sing.

I am the cygnet to this pale faint swan

Who chants a doleful hymn to his own death

And from the organ-pipe of frailty sings

His soul and body to their lasting rest.

Maisc'est évidemment la plongée béatifique et léthale d'Ophélie que cette mort lyrique nous rappelle : le motif végétal et aquatique du saule, le thème récurrent de l'inconstance amoureuse - exprimé par Ophélie sous la forme de bribes de ballades - et l'image subliminale du chant du cygne, créent une parenté isomorphique, que confirme leur statut commun d'objets, non de sujets, tragiques. Si l'eau, selon Bachelard, est la «matière du désespoir», la musique en est l'expression dans cet «espace nul qui s' appelle le temps»70. Par leur caractère ancien ("old lauds") autant que par leur situation dans le drame ${ }^{n}$, ces citations musicales semblent, en arrêtant le cours de l'action, arrêter également celui du temps. 
Dans la vision tragique comme dans l'évocation du merveilleux, la musique est "stase". Elle a dès lors des raisons dramatiques que la raison des mots ne comprend pas. Comment, en effet ne pas lire le "nocturne" de Lorenzo - l'ouïe n'est-elle pas le sens de la nuit? - comme proprement "statique" ? L'attente musicale et de nuit évoque par le discours l'effet même qu'il produit sur son auditoire, unanimement "ému par l'accord de sons harmonieux»" après avoir souffert les affres d'un procès cannibale. «Science des contraires», la musique opère l'union de ce que les mots font divorcer : le monde de l'or et celui de 1'amour. Comme le souligne justement John Hollander ${ }^{73}$, «The Venitian incompatibilities of gold and love are finally reconciled, almost as much in the golden music as in the golden ring". La musique comme "stase" devient une des composantes dramaturgiques, une structure dramatique au sens où la définit Ingram4. La chansón d'Hecate $^{75}$, la musique bruyante de Claudius ${ }^{76}$, 1 'intermède instrumental de Lucius ${ }^{7}$ arrêtent le temps et préparent à la vision. Ainsi la musique abolit-elle les seize ans que la figure du temps a imposés entre l'évanouissement d'Hermione et son réveil's. Ainsi, également, maintient-elle les naufragés de la tempête dans l'illusion bienheureuse du masque de Cérès, où Ferdinand caresse le rêve d'éternité, cependant que le temps continue de s'écouler :

Fer. Let me live here ever's.
Pros. The minute of their plot / Is almost comes.

Cette intemporalité essentielle est marque spécifique de la musique par rapport au langage des mots, car, comme le fait remarquer Boris de Schloezer, «en admettant que la musique organise effectivement le temps, quel est donc le caractère spécifique de cette opération ?... Le compositeur produit dans le temps une chose qui en son unité, en tant qu'ayant un sens, est intemporelle... . ${ }^{81}$. L'intemporalité introduite dans le temps dramatique est le paradoxe essentiel de la représentation musicale, régulièrement associée à des phases intersticielles de la conscience : perte de conscience dans le sommeil (Titania), réveil (Hunt's up, A Midsummer Night's Dream, Pericles), résurrection (Winter's Tale, Pericles), rêve (Catherine, Henry $V I I I^{\prime}$ ), visions infernales ou paradisiaques (Hamlet, Macbeth, Cymbeline, Tempest, As You Like It), démence, plongée mnésique (Lear, Richard II), voire ivresse (le canon serait ainsi la métaphore dramatique de l'ébriété). C'est dans les plages de temps immobile que la musique supplée les mots, déployant sa puissance rétroactive, et contribuant largement au pathétique ou au merveilleux : la mutilation de Lavinia comme le sommeil de Titania, l'angoisse existentielle de Richard II comme le pouvoir magique de Prospero, le désespoir de Desdémone comme celui du Marque d'Hymen sont autant de thèmes dramatiques que la traduction musicale, qu'elle soit allusion métaphorique ou performance scénique, déplace vers l'expression mythique.

Qu'il soit plongée mnésique (dans la tragédie), stase de la vision ou du rêve 
(dans le Masque...) ou simplement emblème du temps cyclique (les «Saisons» d'All's Well, de Twelfth Night et de As You Like It) le matériau musical est indissociablement lié au temps de la durée dramatique - par la signalisation musicale de chaque pièce - mais surtout au temps de la fiction dramatique auquel il donne une cohérence organique.

Pour reprendre les conclusions d'une étude sur les rapports de la musique et de l'imaginaire ${ }^{82}$, on pourra assimiler la musique à une ponctuation dramatique, passage à un niveau de fiction différent, c'est-à-dire, dans la terminologie de la rhétorique, embrayeur. Par ses occurrences non-fonctionnelles autant que par le jeu des sonneries de trompettes, elle est aussi outil dramaturgique et symbolique de la lutte contre le temps. Elle est le matériau non-spatial du passage de la réalité quotidienne à l'illusion dramatique, et d'un niveau de l'illusion à l'autre, que ces représentations «en abîme» prennent la forme d'un masque ( $A Y L, T M P$ ), d'un ballet (TMP, HVIII), d'un mimodrame (Hamlet), d'une pièce dans la pièce (SHR). En rendant ostensibles, ou plutôt audibles, les rouages de son fonctionnement scénique, outil de l'illusion, la musique contribue à se donner une dimension métathéâtrale. Rituel du «décollage» imaginaire, elle tente de domestiquer le passage du temps en «montrant» le temps du passage.

Si donc la musique de scène devient une instance dramatique à part entière dans les pièces tardives, elle le doit bien sûr aux circonstances particulièrement propices du théâtre des Blackfriars et à la vogue croissante des pièces de cour, comme l'a fort bien montré Sternfeld ${ }^{83}$. Mais on ne peut manquer de remarquer que l'évolution générale des mots et de la musique a un certain degré de cohérence paradigmatique, la performance étant commutable avec la référence verbale : la gratuité rhétorique des premières allusions musicales, dans les premières comédies, fait progressivement place à une intégration de plus en plus adéquate de la musique de scène à la trame chronologique et symbolique du drame, où elle résout dans son intemporalité les tensions critiques ; elle est cathartique autant qu'éthique, et répond donc ainsi aux deux exigences qu'Aristote formulait pour la tragédie.

Il est curieux, pour conclure, et sans en induire la moindre relation de causalité immédiate, de constater que la carrière même de Shakespeare est jalonnée par une série de coïncidences musicales (sans parler, bien sûr de ses rencontres avec des musiciens) : ses premières comédies sont contemporaines du madrigal et du ballet, récemment importés d'Italie; les pièces de la maturité sont souvent plus nocturnes, comme les «ayres» de John Dowland, «semper dolens»; les pièces finales sont produites alors que le masque de cour jonsonnien fait figure de modèle. Le mariage parfait de la langue anglaise et de la musique ne survit d'ailleurs guère à l'époque shakespearienne, puisqu'à la fine adéquation du rythme musical au rythme chanté, et à la fidèle restitution du système tonique de la langue dans la métrique musicale, la mode préférera le style plus déclamatoire de l'aria et du récitatif.

Mais une autre caractéristique du drame shakespearien dans ses rapports 
avec la musique est la double approche sémantique qu'ils sous-tendent. On a vu comment les conceptions néo-platoniciennes de Boèce avaient régi la musique spéculative de la Renaissance, et comment la lecture du monde, par le jeu des similitudes était homologiquement organisée en une polysémie toujours triple : phénomène, référent humain, référent universel. Mais si cette allégorie est toujours le moule du discours sur la musique, informant les topoï de l'extase et des effets orphiques, des relations occultes entre proportions musicales et proportions universelles, il est cependant, dès la fin des années 1580 , une tendance nouvelle, clairement explicitée par Thomas Morley dans sa «Plaine and Easie Introduction to Practicall Musicke» (1537) : il s'agit d'un sémantisme de nature également allégorique, mais ayant pour référent unique la passion individuelle. Rythme, accords, intervalles, chromatisme, sont autant d'intonations ou de signes «exprimant» virilité, amertume cruauté :

So that those natural motions may serve to express those effects of cruelty, tyranny, bitterness and such others, and those accidental motions may fitly express the passions of grief, weeping, sighs, sorrows, sobs, and such like $e^{24}$.

On voit donc à quel carrefour de l'herméneutique musicale se situent à la fois un grand nombre de références textuelles à la musique, et un grand nombre de citations proprement musicales dans le drame : vecteur «éthique» de la passion selon des modèles rhétoriques codifiés, voire stéréotypés, ou symbole centripète fondé sur des correspondances. Le vieux débat sur le sémantisme musical, régénéré par les techniques madrigalesques, rejoint alors une problématique plus vaste, qui est celle du langage lui-même : le «word-painting» ne présuppose-t-il pas une théorie du «word»? 


\section{NOTES}

1 Preface to Part I of Rosseter's Ayres, London, 1601 ;rep. in FELLOWES (E.H.). - The English School of Lutenist Song Writers, series I, IV, vii. - London, 1920-32.

2 MORLEY Thomas, A Plaine and Easie Introduction to Practicall Musicke, London, 1597 ; rep. , E.H. Fellowes, ed., in Shakespeare Assocation facsimile, 14, London, 1937, p. 177.

3 Two Gentlemen of Verona, I. ii, 84.

4 The Merry Wives of Windsor II. i, 59-61.

5 Hamlet, V.ii, 184-189.

6 V, v, 23-27.

7 All's Well that Ends Well, III. ii, 8-9.

8 Tempest III. iii, 96-99.

9 CROLLIUS,Traité des Signatures, Trad. française Lyon 1624, pp. 33-34, cité par M. Foucault, Les Mots et les Choses, Paris, Gallimard, 1966, p. 42.

10 The Taming of the Shrew, II. i, 144-149 et III. i, 1-79.

Two Gentlemen of Verona, I. ii, 80-98.

The Tragedy of King Richard the Second, V. v, 41-49.

The Merchant of Venice, V. i, 45-88.

Hamlet, III. ii, 336-363.

11 SCHLOEZER Boris de, Introduction à J.-S. Bach. Essai d' esthétique musicale, Paris, Gallimard, 1947, p. 268.

12 Foucault, Michel, Les Mots et les Choses, Paris, Gallimard, 1966, p. 42.

13 Voir note 9.

14 FOUCAULT Michel, op. cit. (note 12), p. 44.

15 As You Like It II, vii, 139-166.

16 Ibid., II. i, 15-17.

17 The Taming of the Shrew III. i, 74.

18 The Merchant of Venice, V. 00i, 63-65.

19 Timée, 47.

20 FOUCAULT Michel, op. cit, (note 12), p. 44.

21 The Taming of the Shrew, III.i, 11-12.

22 "A Madame Marguerite", Odes, 5ème livre, II.

23 CASTIGLIONE B., The Book of the Courtier done into English by Sir Th Hoby, Amo 1561, edited [...] by W. Raleigh, London, 1900, I, p. 75.

24 Much Ado about Nothing, II. iii, 58-60.

25 Cymbeline, II. iii, 13-29.

26 HERON-ALLEN, Violin-making as it was, an is, London, 1885, pp. 209-210.

27 Twelfth Night, I. i, 1-3.

28 As You Like It, II. v, 11-12. 
29 Ibid., II. $\mathbf{v}, 4$.

30 Ibid., II, vii, 5-6.

31 MERSENNE Marin, Harmonie Universelle, Paris, 1636 ; rep. CNRS, 1975, vol. III, p. 180.

32 Pericles, III.ii, 89-92.

33 MERSENNE Marin, op. cit., note 32, vol. III, p. 30.

34 The Tragedy of King Richard the Third, I. i, 12-13.

35 The First Part of King Henry the Fourth, I. ii, 71-74.

36 The Merchant of Venice, IV. i, 49-50.

37 DU BARTAS, La Sepmaine (1580), Paris, Nizet, 1981, le sixième jour, v. 592-594.

38 Coriolian, I. i, 116.

Winter's Tale, II. ii, 32-35.

39 The Tragedy of King Richard the Third, IV, iv, 364-365.

40 Twelfth Night, V. vii, 23.

41 Pericles, I. ii, 82-86.

42 MARLOWE Christopher, Hero and Leander, I. 229-230.

43 The Tragedy of King Richard the Third, IV. iv, 364-65.

The Famous History of King Henry the Eighth, III. ii, 105-106.

44 The Tragedy of King Richard the Second, I. iii, 161-165, et II. i, 149.

45 Hamlet, Prince of Denmark, III. i, 159-160 (Folio). King Lear, IV, vii 14-17

46 Othello, III. i, 3-11.

47 Hamlet, III. ii, 336-363.

48 The Tragedy of King Richard the Second, I. iii, 159-161.

49 sharp = dièse / pincement ; flat = bémol / gifle :

Two Gentlemen of Verona, I. ii, 90-96:

Lucetta. [...] And methinks I do not like this tune.

Julia. You do not?

Lucetta. No, madam, 'tis too sharp.

Julia. You minion, are too saucy.

Lucetta. Nay, now you are too flat;

And mar the concord with too harsh a descant :

There wanteth but a mean to fill your song.

et The Taming of the Shrew, I. i, 16-17:

Petruchio. Faith, Sirrah, an you'll knock, I'll ring it :

I'll try how you can sol, fa, and sing it.

50 Ibid, II. i, 144-159.

51 Ibid, III. i, 58.

52 Twelfth Night, I. iii, 25-26.

53 The First Part of King Henry the Fourth, III. i, 201-204.

54 The First Part of King Henry the Sixth, IV. i, 188.

55 The Taming of the Shrew, I. ii, 204-209).

56 Antony and Cleopatra, IV, iii, 11 [S.D.] «Music of the hautboys is under the stage».

57 PARTRIDGE Eric, Shakespeare's Bawdy, London, Routledge and Kegan Paul, 1968, "sing", p. 183.

58 Troilus and Cressida, V. iii, 9-12.

59 PARTRIDGE Eric, op. cit. (note 57), «dance with one's heels», p. 91 ; «burden», pp. 
73-74.

60 Much Ado About Nothing, III, iv, 40-45.

61 PARTRIDGE E., op. cit., (note 57), p. 23.

62 MORLEY Thomas, op. cit., (note 2).

63 DURAND Gilbert, Les Structures Anthropologiques de l'Imaginaire, Paris, Dunod, 1969, p. 389.

64 Othello, II. i, 200.

65 BRIL Jacques, «Corps Archaïque, Corps Musical», in La Musique souvent, Essais sur l'imaginaire musical, Recherches et travaux, Université de Grenoble III, Hors série n. 2, 1984, pp. 73-80.

66 Othello, V. ii, 360.

67 Ibid, III. i, 16-17.

68 ISELIN Pierre, «Les Musiques d'Othello», in Autour d'Othello, Sterne, Université de Picardie, 1987, p. 68.

69 The Life and Death of King John, V. vii, 19-24.

70 MICHEL A., Psychanalyse de la musique, Paris, P.U.F., 1956, p. 215.

71 INGRAM R.W., « music as a structural element in Shakespeare», in, World Shakespeare Congress, Vancouver, 1971, pp. 174-189.

72 The Merchant of Venice, V.i, 84.

73 HOLLANDER John, The Untuning of the Sky, Princeton University Press, 1961, p. 153.

74 INGRAM R.W., op. cit., (note 71).

75 Macbeth, IV. i, 43.

76 Hamlet, I. iv, 8-12.

77 Julius Ceasar, IV. iii, $265 \mathrm{ff}$.

78 The Winter's Tale, V. iii, 98.

79 The Tempest, IV. i, 122.

80 Ibid, IV. i, 141-142.

81 SCHLOEZER Boris de, op. cit. (note 11), p. 31.

82 ISELIN Pierre, «Musique et Imaginaire chez William Shahespeare in IRIS, Revue du Centre de Recherches sur l'Imaginaire de Grenoble, n. 2 (4ème trimestre 1986), pp. 26 27.

83 STERNFELD F.W., Music in Shakespearean Tragedy, N.Y., Dover Publications, 1963.

84 MORLEY Thomas, op.cit., p. 177. 